\title{
Monotonicity and inequalities for the gamma function
}

Zhen-Hang Yang ${ }^{1,2}$ and Jing-Feng Tian ${ }^{{ }^{*}}$

\section{${ }^{*}$ Correspondence:}

tianjf@ncepu.edu.cn

${ }^{1}$ College of Science and

Technology, North China Electric

Power University, Ruixiang Street

282, Baoding, 071051, P.R. China

Full list of author information is

available at the end of the article

\section{Abstract}

In this paper, by using the monotonicity rule for the ratio of two Laplace transforms, we prove that the function

$$
x \mapsto \frac{1}{24 x(\ln \Gamma(x+1 / 2)-x \ln x+x-\ln \sqrt{2 \pi})+1}-\frac{120}{7} x^{2}
$$

is strictly increasing from $(0, \infty)$ onto $(1,1860 / 343)$. This not only yields some known and new inequalities for the gamma function, but also gives some completely monotonic functions related to the gamma function.

MSC: Primary 33B15; 26A48; secondary 26D15; 26A51

Keywords: gamma function; Laplace transform; complete monotonicity; inequality

\section{Introduction}

Stirling's formula

$$
n ! \sim \sqrt{2 \pi n} n^{n} e^{-n}
$$

has important applications in statistical physics, probability theory and number theory. Due to its practical importance, it has attracted much interest of many mathematicians and have motivated a large number of research papers concerning various generalizations and improvements.

Burnside's formula [1]

$$
n ! \sim \sqrt{2 \pi}\left(\frac{n+1 / 2}{e}\right)^{n+1 / 2}:=b_{n}
$$

slightly improves (1.1). Gosper [2] replaced $\sqrt{2 \pi n}$ by $\sqrt{2 \pi(n+1 / 6)}$ in (1.1) to get

$$
n ! \sim \sqrt{2 \pi\left(n+\frac{1}{6}\right)}\left(\frac{n}{e}\right)^{n}:=g_{n}
$$

(c) The Author(s) 2017. This article is distributed under the terms of the Creative Commons Attribution 4.0 International License (http://creativecommons.org/licenses/by/4.0/), which permits unrestricted use, distribution, and reproduction in any medium, provided you give appropriate credit to the original author(s) and the source, provide a link to the Creative Commons license, and indicate if changes were made. 
which is better than (1.1) and (1.2). Batir [3] obtained an asymptotic formula similar to (1.3):

$$
n ! \sim \frac{n^{n+1} e^{-n} \sqrt{2 \pi}}{\sqrt{n-1 / 6}}:=b_{n}^{\prime}
$$

which is stronger than (1.1) and (1.2). A more accurate approximation for the factorial function

$$
n ! \sim \sqrt{2 \pi}\left(\frac{n^{2}+n+1 / 6}{e^{2}}\right)^{n / 2+1 / 4}:=m_{n}
$$

was presented in [4] by Mortici.

The gamma function $\Gamma(x)=\int_{0}^{\infty} t^{x-1} e^{-t} d t$ for $x>0$ is closely related to Stirling's formula since $\Gamma(n+1)=n$ ! for all $n \in \mathbb{N}$. This inspires some authors to also pay attention to finding various better approximations for the gamma function. Here we list some more accurate approximations:

(i) Ramanujan's [5, p. 339] approximation formula as $x \rightarrow \infty$

$$
\Gamma(x+1) \sim \sqrt{\pi}\left(\frac{x}{e}\right)^{x}\left(8 x^{3}+4 x^{2}+x+\frac{1}{30}\right)^{1 / 6}:=R(x) ;
$$

(ii) Windschitl's (see [6, Eq. (42)]) approximation formula

$$
\Gamma(x+1) \sim \sqrt{2 \pi x}\left(\frac{x}{e}\right)^{x}\left(x \sinh \frac{1}{x}\right)^{x / 2}:=W(x)
$$

(iii) Smith's [6, Eq. (42)] approximation formula

$$
\Gamma\left(x+\frac{1}{2}\right) \sim \sqrt{2 \pi}\left(\frac{x}{e}\right)^{x}\left(2 x \tanh \frac{1}{2 x}\right)^{x / 2}:=S(x)
$$

(iv) Nemes' formula ([7, Corollary 4.1]) states that

$$
\Gamma(x+1) \sim \sqrt{2 \pi x}\left(\frac{x}{e}\right)^{x}\left(1+\frac{1}{12 x^{2}-1 / 10}\right)^{x}:=N(x) ;
$$

(v) Chen's [8] presented a new approximation

$$
\Gamma(x+1) \sim \sqrt{2 \pi x}\left(\frac{x}{e}\right)^{x}\left(1+\frac{1}{12 x^{3}+24 x / 7-1 / 2}\right)^{x^{2}+53 / 210}=C(x) .
$$

Remark 1 Let $A(x)$ be an approximation for $\Gamma(x+1)$ as $x \rightarrow \infty$. If there is $m>0$ such that

$$
\lim _{x \rightarrow \infty} \frac{\ln \Gamma(x+1)-\ln A(x)}{x^{-m}}=c \neq 0, \pm \infty
$$

then we say that the rate of $A(x)$ converging to $\Gamma(x+1)$ is like $x^{-m}$ as $x \rightarrow \infty$. Evidently, the larger $m$ is, the higher the accuracy of $A(x)$ approximating for $\Gamma(x+1)$ is. Since $(x-$ 
1)/ $\ln x \rightarrow 1$ as $x \rightarrow 1$, the limit relation can be equivalently written as

$$
\lim _{x \rightarrow \infty} \frac{\Gamma(x+1) / A(x)-1}{x^{-m}}=c \neq 0, \pm \infty,
$$

or

$$
\frac{\Gamma(x+1)}{A(x)}=1+O\left(x^{-m}\right) \quad \text { as } x \rightarrow \infty .
$$

Remark 2 It is easy to check that as $n \rightarrow \infty$ or $x \rightarrow \infty$,

$$
\begin{aligned}
& n !=\frac{n^{n+1} e^{-n} \sqrt{2 \pi}}{\sqrt{n-1 / 6}}\left(1+O\left(n^{-2}\right)\right), \\
& n !=\sqrt{2 \pi}\left(\frac{n^{2}+n+1 / 6}{e^{2}}\right)^{n / 2+1 / 4}\left(1+O\left(n^{-3}\right)\right), \\
& \Gamma\left(x+\frac{1}{2}\right)=\sqrt{2 \pi}\left(\frac{x}{e}\right)^{x}\left(2 x \tanh \frac{1}{2 x}\right)^{x / 2}\left(1+O\left(x^{-5}\right)\right) .
\end{aligned}
$$

These together with those shown in $[8,(3.5)-(3.10)]$ indicate that Chen's one $C(x)$ is the best among approximation formulas listed above.

More results involving the approximation formulas for the factorial or gamma function can be found in [9-28] and the references cited therein.

It is worth mentioning that Yang and Chu [9] proposed a new approach to construct asymptotic formulas by bivariate means. As applications, they offered in [9, Propositions 4 and 5] two asymptotic formulas: as $x \rightarrow \infty$,

$$
\begin{aligned}
& \Gamma(x+1) \sim \sqrt{2 \pi}\left(\frac{x+1 / 2}{e}\right)^{x+1 / 2} \exp \left(-\frac{1}{24} \frac{x+1 / 2}{x^{2}+x+37 / 120}\right):=Y_{1}(x), \\
& \Gamma(x+1) \sim \sqrt{2 \pi}\left(\frac{x+1 / 2}{e}\right)^{x+1 / 2} \exp \left(-\frac{1517}{44,640} \frac{1}{x+1 / 2}-\frac{343}{44,640} \frac{x+1 / 2}{x^{2}+x+111 / 196}\right) \\
&:=Y_{2}(x),
\end{aligned}
$$

which satisfy

$$
\Gamma(x+1)=Y_{1}(x)\left(1+O\left(x^{-5}\right)\right) \quad \text { and } \quad \Gamma(x+1)=Y_{2}(x)\left(1+O\left(x^{-7}\right)\right)
$$

and proved that the functions (replace $x$ by $x-1 / 2$ )

$$
\begin{aligned}
& f_{4}\left(x-\frac{1}{2}\right)=\ln \Gamma\left(x+\frac{1}{2}\right)-\frac{1}{2} \ln 2 \pi-x \ln x+x+\frac{1}{24} \frac{x}{x^{2}+7 / 120} \\
& f_{5}\left(x-\frac{1}{2}\right)=\ln \Gamma\left(x+\frac{1}{2}\right)-\frac{1}{2} \ln 2 \pi-x \ln x+\frac{1}{1440} \frac{5880 x^{2}+1517}{x\left(98 x^{2}+31\right)}
\end{aligned}
$$

are increasingly concave and decreasingly convex on $(0, \infty)$, respectively. Clearly, both $Y_{1}(x)$ and $Y_{2}(x)$ are accurate and simpler approximation formulas for the gamma function. 
According to these inequalities given in [9, Corollary 7], it is natural to ask: What are the best $\alpha$ and $\beta$ such that the double inequality

$$
\exp \left[-\frac{1}{24 x} \frac{120 x^{2}+7(\alpha-1)}{120 x^{2}+7 \alpha}\right]<\frac{\Gamma(x+1 / 2)}{\sqrt{2 \pi}(x / e)^{x}}<\exp \left[-\frac{1}{24 x} \frac{120 x^{2}+7(\beta-1)}{120 x^{2}+7 \beta}\right]
$$

holds for all $x>0$ ? This problem is equivalent to determining the monotonicity of the function

$$
f(x)=\frac{1}{24 x(\ln \Gamma(x+1 / 2)-x \ln x+x-\ln \sqrt{2 \pi})+1}-\frac{120}{7} x^{2}
$$

on $(0, \infty)$.

The aim of this paper is to answer this problem. Our main result is the following theorem.

Theorem 1 The function $f$ defined by (1.13) is strictly increasing from $(0, \infty)$ onto $(1,1860 / 343)$.

As a consequence of the above theorem, the following corollary is immediate.

Corollary 1 For $x>x_{0} \geq 0$, the double inequality (1.12) holds if and only if $\alpha \geq f(\infty)=$ $1860 / 343$ and $1 \leq \beta \leq f\left(x_{0}\right)$. In particular, we have

$$
\exp \left[-\frac{1}{1440} \frac{5880 x^{2}+1517}{x\left(98 x^{2}+31\right)}\right]<\frac{\Gamma(x+1 / 2)}{\sqrt{2 \pi}(x / e)^{x}}<\exp \left(-\frac{5 x}{120 x^{2}+7}\right)
$$

holds for $x>0$.

Replacing $x$ by $n+1 / 2$, then putting $x_{0}=1$ in Corollary 1 , and noting that

$$
\beta_{1}:=f\left(\frac{3}{2}\right)=\frac{1}{36 \ln 2-54 \ln 3-18 \ln \pi+55}-\frac{270}{7} \approx 4.7243,
$$

we deduce the following statement.

Corollary 2 The double inequality

$$
\begin{aligned}
& \exp \left[-\frac{1}{24(n+1 / 2)} \frac{120(n+1 / 2)^{2}+7(\alpha-1)}{120(n+1 / 2)^{2}+7 \alpha}\right] \\
& <\frac{n !}{\sqrt{2 \pi}((n+1 / 2) / e)^{n+1 / 2}} \\
& \quad<\exp \left[-\frac{1}{24(n+1 / 2)} \frac{120(n+1 / 2)^{2}+7\left(\beta_{1}-1\right)}{120(n+1 / 2)^{2}+7 \beta_{1}}\right]
\end{aligned}
$$

holds with the best constants $\beta_{1} \approx 4.7243$ given by (1.14) and $\alpha=1860 / 343 \approx 5.4227$. 


\section{Tools}

To prove our main result, we need some lemmas as tools. The first lemma is the convolution formula of the Laplace transform.

Lemma 1 ([29]) Let $f_{i}(t)$ for $i=1,2$ be piecewise continuous in arbitrary finite intervals included on $(0, \infty)$. If there exist some constants $M_{i}>0$ and $c_{i} \geq 0$ such that $\left|f_{i}(t)\right| \leq M_{i} e^{c_{i} t}$ for $i=1,2$, then

$$
\int_{0}^{\infty} f_{1}(u) e^{-s u} d u \int_{0}^{\infty} f_{2}(v) e^{-s v} d v=\int_{0}^{\infty}\left(\int_{0}^{t} f_{1}(u) f_{2}(t-u) d u\right) e^{-s t} d t
$$

The second one is a special monotonicity rule for the ratio of two power series, which first appeared in [30, Lemma 6.4] and was proved in [31], also see [32].

Lemma 2 ([31, Corollary 2.3]) Let $A(t)=\sum_{k=0}^{\infty} a_{k} t^{k}$ and $B(t)=\sum_{k=0}^{\infty} b_{k} t^{k}$ be two real power series converging on $\mathbb{R}$ with $b_{k}>0$ for all $k$. If, for certain $m \in \mathbb{N}$, the sequence $\left\{a_{k} / b_{k}\right\}$ is increasing (decreasing) for $0 \leq k \leq m$ and decreasing (increasing) for $k \geq m$, then there is a unique $t_{0} \in(0, \infty)$ such that the function $A / B$ is increasing (decreasing) on $\left(0, t_{0}\right)$ and decreasing (increasing) on $\left(t_{0}, \infty\right)$.

The third lemma is called L'Hospital piecewise monotonicity rule [33].

Lemma 3 ([33, Theorem 8]) Let $-\infty \leq a<b \leq \infty$. Suppose that (i) $f$ and $g$ are differentiable functions on $(a, b)$; (ii) $g^{\prime} \neq 0$ on $(a, b)$; (iii) $f\left(a^{+}\right)=g\left(a^{+}\right)=0$; (iv) there is $c \in(a, b)$ such that $f^{\prime} / g^{\prime}$ is increasing (decreasing) on ( $\left.a, c\right)$ and decreasing (increasing) on $(c, b)$. Then

(i) when $\operatorname{sgn} g^{\prime} \operatorname{sgn} H_{f, g}\left(b^{-}\right) \geq(\leq) 0, f / g$ is increasing (decreasing) on $(a, b)$, where $H_{f, g}=\left(f^{\prime} / g^{\prime}\right) g-f$

(ii) when $\operatorname{sgn} g^{\prime} \operatorname{sgn} H_{f, g}\left(b^{-}\right)<(>) 0$, there is a unique number $x_{a} \in(a, b)$ such that $f / g$ is increasing (decreasing) on $\left(a, x_{a}\right)$ and decreasing (increasing) on $\left(x_{a}, b\right)$.

The last one gives a monotonicity rule for the ratio of two Laplace transforms, which is crucial to proving our main result (see [34, Remark 3]).

Lemma 4 Let the functions $A, B$ be defined on $(0, \infty)$ such that their Laplace transforms exist with $B(t) \neq 0$ for all $t>0$. Then the function

$$
x \mapsto U(x)=\frac{\int_{0}^{\infty} A(t) e^{-x t} d t}{\int_{0}^{\infty} B(t) e^{-x t} d t}
$$

is decreasing (increasing) on $(0, \infty)$ if $A / B$ is increasing (decreasing) on $(0, \infty)$.

Proof Differentiation yields

$$
\begin{aligned}
& \left(\int_{0}^{\infty} B(t) e^{-x t} d t\right)^{2} U^{\prime}(x) \\
& \quad=-\int_{0}^{\infty} A(t) e^{-x t} d t \int_{0}^{\infty} B(t) e^{-x t} d t+\int_{0}^{\infty} A(t) e^{-x t} d t \int_{0}^{\infty} t B(t) e^{-x t} d t \\
& \quad=\int_{0}^{\infty} \int_{0}^{\infty} t\left[\frac{A(s)}{B(s)}-\frac{A(t)}{B(t)}\right] B(t) B(s) e^{-x t-x s} d s d t:=D .
\end{aligned}
$$


Exchanging the integral variables $s$ and $t$, we have

$$
D=\int_{0}^{\infty} \int_{0}^{\infty} s\left[\frac{A(t)}{B(t)}-\frac{A(s)}{B(s)}\right] B(s) B(t) e^{-x s-x t} d t d s
$$

then adding gives

$$
2 D=-\int_{0}^{\infty} \int_{0}^{\infty}[t-s]\left[\frac{A(t)}{B(t)}-\frac{A(s)}{B(s)}\right] B(s) B(t) e^{-x t-x s} d t d s
$$

By the assumptions, the desired assertions follow.

\section{Proof of Theorem 1}

Before proving Theorem 1, we also need several concrete lemmas.

Lemma 5 ([28, Lemma 4]) Let $g_{0}$ be defined on $(0, \infty)$ by

$$
g_{0}(x)=\ln \Gamma\left(x+\frac{1}{2}\right)-x \ln x+x-\frac{1}{2} \ln (2 \pi)
$$

Then $g_{0}(x)$ has the following integral representation:

$$
g_{0}(x)=-\int_{0}^{\infty} h(t) e^{-x t} d t
$$

where

$$
h(t)=\frac{1}{t^{2}}-\frac{1}{2 t \sinh (t / 2)} .
$$

Lemma 6 Let $h(t)$ be defined on $(0, \infty)$ by (3.3). Then we have

$$
\begin{aligned}
& x \int_{0}^{\infty} h(t) e^{-x t} d t=\frac{1}{24}+\int_{0}^{\infty} h^{\prime}(t) e^{-x t} d t, \\
& x \int_{0}^{\infty} h^{\prime}(t) e^{-x t} d t=\int_{0}^{\infty} h^{\prime \prime}(t) e^{-x t} d t, \\
& x \int_{0}^{\infty} h^{\prime \prime}(t) e^{-x t} d t=-\frac{7}{2880}+\int_{0}^{\infty} h^{\prime \prime \prime}(t) e^{-x t} d t .
\end{aligned}
$$

Proof Integration by parts yields

$$
x \int_{0}^{\infty} h(t) e^{-x t} d t=-\int_{0}^{\infty} h(t) d e^{-x t}=-\left[h(t) e^{-x t}\right]_{t=0}^{t=\infty}+\int_{0}^{\infty} h^{\prime}(t) e^{-x t} d t
$$

which, by a simple computation,

$$
\begin{aligned}
& \lim _{t \rightarrow 0} h(t) e^{-x t}=\lim _{t \rightarrow 0}\left(\frac{1}{t^{2}}-\frac{1}{2 t \sinh (t / 2)}\right) e^{-x t}=\frac{1}{24}, \\
& \lim _{t \rightarrow \infty} h(t) e^{-x t}=\lim _{t \rightarrow \infty}\left(\frac{1}{t^{2}}-\frac{1}{2 t \sinh (t / 2)}\right) e^{-x t}=0,
\end{aligned}
$$

gives (3.4). 
Similarly, integration by parts and limit relations $\lim _{t \rightarrow 0} h^{\prime}(t) e^{-x t}=0$ and $\lim _{t \rightarrow \infty} h^{\prime}(t) e^{-x t}=0$ yield (3.5). Integration by parts in combination with $\lim _{t \rightarrow 0} h^{\prime \prime}(t) e^{-x t}=$ $-7 / 2880$ and $\lim _{t \rightarrow \infty} h^{\prime \prime}(t) e^{-x t}=0$ gives (3.6).

Lemma 7 Let $h(t)$ be defined by (3.3). Then (i) $h^{\prime}(t)<0$ for $t>0$; (ii) there is $t_{0}>0$ such that the function $h^{\prime \prime \prime} / h^{\prime}$ is increasing on $\left(0, t_{0}\right)$ and decreasing on $\left(t_{0}, \infty\right)$. Therefore, we have

$$
-\frac{31}{98}<\frac{h^{\prime \prime \prime}(t)}{h^{\prime}(t)}<\lambda_{0} \approx 0.051704
$$

where $\lambda_{0}=h^{\prime \prime \prime}\left(t_{0}\right) / h^{\prime}\left(t_{0}\right)$, here $t_{0}$ is the unique solution of the equation $\left[h^{\prime \prime \prime}(t) / h^{\prime}(t)\right]^{\prime}=0$ on $(0, \infty)$

Proof Differentiation yields

$$
\begin{aligned}
h^{\prime}(t)= & \frac{1}{4} \frac{2 \sinh (t / 2)+t \cosh (t / 2)}{t^{2} \sinh ^{2}(t / 2)}-\frac{2}{t^{3}}, \\
h^{\prime \prime}(t)= & \frac{6}{t^{4}}-\frac{1}{16} \frac{t^{2} \cosh t+8 \cosh t+4 t \sinh t+3 t^{2}-8}{t^{3} \sinh ^{3}(t / 2)} \\
h^{\prime \prime \prime}(t)= & -\frac{24}{t^{5}}+\frac{1}{64 t^{4} \sinh ^{4}(t / 2)}\left(6 t^{2} \sinh \frac{3 t}{2}+48 \sinh \frac{3 t}{2}+t^{3} \cosh \frac{3 t}{2}+24 t \cosh \frac{3 t}{2}\right. \\
& \left.+23 t^{3} \cosh \frac{t}{2}-24 t \cosh \frac{t}{2}+30 t^{2} \sinh \frac{t}{2}-144 \sinh \frac{t}{2}\right) .
\end{aligned}
$$

Simplifying and expanding in power series yield

$$
\begin{aligned}
-\left(4 t^{3} \sinh ^{2} \frac{t}{2}\right) h^{\prime}(t) & =4 \cosh t-t^{2} \cosh \frac{t}{2}-2 t \sinh \frac{t}{2}-4 \\
& =\sum_{n=3}^{\infty} \frac{2^{2 n-2}-n^{2}}{2^{2 n-4}(2 n) !} t^{2 n}>0
\end{aligned}
$$

which proves $h^{\prime}(t)<0$ for $t>0$.

Then $h^{\prime \prime \prime}(t) / h^{\prime}(t)$ can be expressed as

$$
\begin{aligned}
\frac{h^{\prime \prime \prime}(t)}{h^{\prime}(t)}= & \frac{96 \sinh ^{4} s-3 s^{3} \sinh 3 s-6 s \sinh 3 s-s^{4} \cosh 3 s-6 s^{2} \cosh 3 s}{16 s^{2}\left(2 \sinh ^{2} s-s \sinh s-s^{2} \cosh s\right) \sinh ^{2} s} \\
& +\frac{-15 s^{3} \sinh s+18 s \sinh s-23 s^{4} \cosh s+6 s^{2} \cosh s}{16 s^{2}\left(2 \sinh ^{2} s-s \sinh s-s^{2} \cosh s\right) \sinh ^{2} s}:=\frac{h_{1}(s)}{h_{2}(s)}
\end{aligned}
$$

where $s=2 t$. Using 'product into sum' formula for hyperbolic functions and expanding in power series give

$$
\begin{aligned}
h_{1}(s):= & 12 \cosh 4 s-48 \cosh 2 s-3 s^{3} \sinh 3 s-6 s \sinh 3 s-s^{4} \cosh 3 s-6 s^{2} \cosh 3 s \\
& -15 s^{3} \sinh s+18 s \sinh s-23 s^{4} \cosh s+6 s^{2} \cosh s+36 \\
= & 12 \sum_{n=0}^{\infty} \frac{4^{2 n}}{(2 n) !} s^{2 n}-48 \sum_{n=0}^{\infty} \frac{2^{2 n}}{(2 n) !} s^{2 n}-3 \sum_{n=2}^{\infty} \frac{3^{2 n-3}}{(2 n-3) !} s^{2 n}
\end{aligned}
$$




$$
\begin{aligned}
& \quad-6 \sum_{n=1}^{\infty} \frac{3^{2 n-1}}{(2 n-1) !} s^{2 n}-\sum_{n=2}^{\infty} \frac{3^{2 n-4}}{(2 n-4) !} s^{2 n}-6 \sum_{n=1}^{\infty} \frac{3^{2 n-2}}{(2 n-2) !} s^{2 n}-15 \sum_{n=2}^{\infty} \frac{1}{(2 n-3) !} s^{2 n} \\
& \quad+18 \sum_{n=1}^{\infty} \frac{1}{(2 n-1) !} s^{2 n}-23 \sum_{n=2}^{\infty} \frac{1}{(2 n-4) !} s^{2 n}+6 \sum_{n=1}^{\infty} \frac{1}{(2 n-2) !} s^{2 n} \\
& :=4 \sum_{n=2}^{\infty} \frac{a_{n}}{(2 n) !} s^{2 n},
\end{aligned}
$$

where

$$
\begin{aligned}
a_{n}=3 & \times 4^{2 n}-12 \times 2^{2 n}-2 n\left(2 n^{3}+3 n^{2}+19 n+30\right) 3^{2 n-4} \\
- & 2 n(2 n-3)\left(23 n^{2}-27 n+10\right), \\
h_{2}(s): & =16 s^{2}\left(2 \sinh ^{2} s-s \sinh s-s^{2} \cosh s\right) \sinh ^{2} s \\
= & 4 s^{2}\left(\cosh 4 s-4 \cosh 2 s-s \sinh 3 s-s^{2} \cosh 3 s+s^{2} \cosh s+3 s \sinh s+3\right) \\
= & 4 s^{2}\left(\sum_{n=1}^{\infty} \frac{4^{2 n}}{(2 n) !} s^{2 n}-4 \sum_{n=1}^{\infty} \frac{2^{2 n}}{(2 n) !} s^{2 n}-\sum_{n=1}^{\infty} \frac{3^{2 n-1}}{(2 n-1) !} s^{2 n}-\sum_{n=1}^{\infty} \frac{3^{2 n-2}}{(2 n-2) !} s^{2 n}\right. \\
& \left.+\sum_{n=1}^{\infty} \frac{1}{(2 n-2) !} s^{2 n}+3 \sum_{n=1}^{\infty} \frac{1}{(2 n-1) !} s^{2 n}+3\right):=4 \sum_{n=2}^{\infty} \frac{b_{n}}{(2 n) !} s^{2 n},
\end{aligned}
$$

where

$$
b_{n}=2 n(2 n-1)\left(4^{2 n-2}-2^{2 n}-4 n(n-1) 3^{2 n-4}+4 n(n-1)\right) .
$$

Thus, if we prove the sequence $\left\{a_{n} / b_{n}\right\}_{n \geq 5}$ is increasing then decreasing, then by Lemma 2 we deduce that there is $t_{0}$ such that $h^{\prime \prime} / h$ is increasing on $\left(0, t_{0}\right)$ and decreasing on $\left(t_{0}, \infty\right)$, and the proof is done. To this end, if $b_{n}>0$ for $n \geq 5$, then it suffices to show that there is $n_{0}>5$ such that $d_{n}=a_{n} b_{n+1}-b_{n} a_{n+1} \leq 0$ for $5 \leq n \leq n_{0}$ and $d_{n} \geq 0$ for $n \geq n_{0}$.

Now, it is easy to check that

$$
\frac{b_{n+1}}{2(n+1)(2 n+1)}-16 \frac{b_{n}}{2 n(2 n-1)}=4 n(7 n-25) 3^{2 n-4}+12 \times 2^{2 n}-4 n(15 n-17)>0,
$$

which together with $b_{4}=0$ yields $b_{n}>0$ for $n \geq 5$. On the other hand, by an elementary computation, we obtain

$$
d_{n}=a_{n} b_{n+1}-b_{n} a_{n+1}=\sum_{k=1,2,3,4,6,8,9,12,16} p_{k}(n) k^{2 n},
$$

where

$$
\begin{aligned}
& p_{16}(n)=6(4 n+1), \\
& p_{12}(n)=-\frac{1}{324} n\left(28 n^{5}+12 n^{4}-1181 n^{3}+9678 n^{2}+3457 n+1830\right), \\
& p_{9}(n)=\frac{64}{243} n^{2}(n+1)\left(n^{3}+8 n^{2}+20 n-2\right),
\end{aligned}
$$




$$
\begin{aligned}
& p_{8}(n)=6\left(18 n^{2}-41 n-8\right), \\
& p_{6}(n)=-\frac{4}{81} n\left(20 n^{5}+132 n^{4}+185 n^{3}-678 n^{2}-997 n-822\right), \\
& p_{4}(n)=-\frac{1}{4}\left(1380 n^{6}-1804 n^{5}+989 n^{4}-3134 n^{3}+1327 n^{2}-2118 n-384\right), \\
& p_{3}(n)=\frac{128}{27} n^{2}(n+1)\left(32 n^{5}-32 n^{4}-33 n^{3}+48 n^{2}-50 n+8\right), \\
& p_{2}(n)=4 n\left(276 n^{5}-508 n^{4}-295 n^{3}+106 n^{2}+43 n-150\right), \\
& p_{1}(n)=192 n^{2}(n+1)\left(9 n^{3}-8 n^{2}+2\right) .
\end{aligned}
$$

An easy verification yields

$$
\begin{aligned}
& d_{5}=-4,007,555,481,600, \\
& d_{6}=-3,910,448,396,574,720, \\
& d_{7}=-1,900,746,298,639,319,040, \\
& d_{8}=-630,125,315,460,849,991,680, \\
& d_{9}=-150,180,694,294,194,463,408,128, \\
& d_{10}=-20,155,436,802,005,011,207,151,616,
\end{aligned}
$$

and $d_{11}=3,463,285,943,229,784,738,339,553,280>0$. It remains to show $d_{n}>0$ for $n \geq 11$. To this end, we write $d_{n}$ as

$$
\begin{aligned}
d_{n}= & {\left[p_{16}(n) \times 16^{2 n}+p_{12}(n) \times 12^{2 n}\right] } \\
& +\left[p_{9}(n) \times 9^{2 n}+p_{6}(n) \times 6^{2 n}\right]+\left[p_{8}(n) \times 8^{2 n}+p_{4}(n) \times 4^{2 n}\right] \\
& +\left[p_{3}(n) \times 3^{2 n}+p_{2}(n) \times 2^{2 n}+p_{1}(n)\right],
\end{aligned}
$$

and denote the expressions in the square brackets by $d_{n}^{\prime}, d_{n}^{\prime \prime}, d_{n}^{\prime \prime \prime}$ and $d_{n}^{\prime \prime \prime \prime}$, respectively. We easily get the recurrence relation of $d_{n}^{\prime}$

$$
\begin{aligned}
\frac{p_{16}(n) d_{n+1}^{\prime}-16^{2} p_{16}(n+1) d_{n}^{\prime}}{12^{2 n}} \\
=144 p_{16}(n) p_{12}(n+1)-16^{2} p_{16}(n+1) p_{12}(n) \\
=\frac{8}{27}\left(784 n^{7}-3724 n^{6}-51,008 n^{5}+328,397 n^{4}+10,762 n^{3}\right. \\
\left.\quad-1,037,977 n^{2}-650,802 n-124,416\right) \\
=\frac{8}{27}\left(784 m^{7}+23,716 m^{6}+248,872 m^{5}+1,086,697 m^{4}+1,666,702 m^{3}\right. \\
\left.\quad+1,160,503 m^{2}+10,500,078 m+20,928,024\right)>0,
\end{aligned}
$$

where $m=n-5 \geq 6$. This in combination with $p_{16}(n)>0$ and $d_{11}^{\prime}=2^{45} \times$ $71,481,197,516,733>0$ leads us to $d_{n}^{\prime}>0$ for $n \geq 11$. 
Similarly, we have

$$
\begin{aligned}
\frac{p_{9}(n) d_{n+1}^{\prime \prime}-81 p_{9}(n+1) d_{n}^{\prime \prime}}{6^{2 n}} & \\
= & 36 p_{9}(n) p_{6}(n+1)-81 p_{9}(n+1) p_{6}(n) \\
= & \frac{256}{2187} n(n+1)^{2}\left(100 n^{9}+1960 n^{8}+15,413 n^{7}+55,819 n^{6}+53,414 n^{5}\right. \\
& \left.-273,428 n^{4}-1,024,655 n^{3}-1,559,511 n^{2}-1,278,612 n-399,492\right)>0
\end{aligned}
$$

for $n \geq 3$. This together with $p_{9}(n)>0$ and $d_{3}^{\prime \prime}=717,610,752>0$ yields $d_{n}^{\prime \prime}>0$ for $n \geq 3$.

Also, we get

$$
\begin{aligned}
\frac{p_{8}(n) d_{n+1}^{\prime \prime \prime}-64 p_{8}(n+1) d_{n}^{\prime \prime \prime}}{4^{2 n}} & \\
= & 16 p_{8}(n) p_{4}(n+1)-64 p_{8}(n+1) p_{4}(n) \\
= & 1,788,480 n^{8}-5,219,424 n^{7}-367,632 n^{6}+8,703,096 n^{5}+13,278,240 n^{4} \\
& \quad+9,974,760 n^{3}-7,438,608 n^{2}+1,718,592 n+423,936
\end{aligned}
$$

which can be rewritten as

$$
\begin{aligned}
& 1,788,480 m^{8}+23,396,256 m^{7}+126,870,192 m^{6}+367,098,936 m^{5}+619,910,160 m^{4} \\
& +687,582,120 m^{3}+676,606,944 m^{2}+635,328,864 m+311,091,840>0,
\end{aligned}
$$

where $m=n-2 \geq 9$. This in combination with $p_{8}(n)>0$ for $n \geq 3$ and $d_{7}^{\prime \prime \prime}=2^{30} \times$ $6,089,535>0$ indicates that $d_{n}^{\prime \prime \prime}>0$ for $n \geq 7$.

As far as $d_{n}^{\prime \prime \prime \prime}>0$ for $n \geq 11$, it is clear, since

$$
\begin{aligned}
\frac{27}{128 n^{2}(n+1)} p_{3}(n) & =\left(32 n^{5}-32 n^{4}-33 n^{3}+48 n^{2}-50 n+8\right) \\
& =32 m^{5}+288 m^{4}+991 m^{3}+1642 m^{2}+1282 m+348>0,
\end{aligned}
$$

where $m=n-2>0$,

$$
\begin{aligned}
\frac{p_{2}(n)}{4 n} & =\left(276 n^{5}-508 n^{4}-295 n^{3}+106 n^{2}+43 n-150\right) \\
& =276 m^{5}+3632 m^{4}+18,449 m^{3}+44,539 m^{2}+49,630 m+18,888>0
\end{aligned}
$$

for $m=n-3>0, p_{1}(n)=192 n^{2}(n+1)\left(9 n^{3}-8 n^{2}+2\right)>0$ for $n \geq 1$. This proves the piecewise monotonicity of $h^{\prime \prime \prime} / h^{\prime}$ on $(0, \infty)$.

It is easy to verify that

$$
\lim _{t \rightarrow 0} \frac{h^{\prime \prime \prime}(t)}{h^{\prime}(t)}=-\frac{31}{98} \quad \text { and } \quad \lim _{t \rightarrow \infty} \frac{h^{\prime \prime \prime}(t)}{h^{\prime}(t)}=0 .
$$

Solving the equation $\left[h^{\prime \prime \prime}(t) / h^{\prime}(t)\right]^{\prime}=0$ yields $t=t_{0} \approx 10.96011$, which gives $\lambda_{0}=h^{\prime \prime \prime}\left(t_{0}\right) /$ $h^{\prime}\left(t_{0}\right) \approx 0.051704$. 
By the piecewise monotonicity of $h^{\prime \prime \prime} / h^{\prime}$ on $(0, \infty)$, we conclude that

$$
-\frac{7}{120}=\min \left(\lim _{t \rightarrow 0} \frac{h^{\prime \prime \prime}(t)}{h^{\prime}(t)}, \lim _{t \rightarrow \infty} \frac{h^{\prime \prime \prime}(t)}{h^{\prime}(t)}\right)<\frac{h^{\prime \prime \prime}(t)}{h^{\prime}(t)}<\frac{h^{\prime \prime \prime}\left(t_{0}\right)}{h^{\prime}\left(t_{0}\right)}=\lambda_{0} \approx 0.051704
$$

which completes the proof.

We now are in a position to prove Theorem 1.

Proof of Theorem 1 We first prove that

$$
f(x)=-\frac{1}{168} \frac{\int_{0}^{\infty}\left(7+2880 h^{\prime \prime}(t)\right) e^{-x t} d t}{\int_{0}^{\infty}\left(\int_{0}^{t} h^{\prime}(s) d s\right) e^{-x t} d t}:=-\frac{1}{168} \frac{\int_{0}^{\infty} A(t) e^{-x t} d t}{\int_{0}^{\infty} B(t) e^{-x t} d t}
$$

where

$$
A(t)=7+2880 h^{\prime \prime}(t) \text { and } B(t)=\int_{0}^{t} h^{\prime}(s) d s
$$

In fact, by Lemma 5 and identities (3.4) and (3.5), $f(x)$ can be expressed as

$$
\begin{aligned}
f(x) & =-\frac{1}{24 \int_{0}^{\infty} h^{\prime}(t) e^{-x t} d t}-\frac{120}{7} x^{2} \\
& =-\frac{7+2880 x^{2} \int_{0}^{\infty} h^{\prime}(t) e^{-x t} d t}{168 \int_{0}^{\infty} h^{\prime}(t) e^{-x t} d t}=-\frac{1}{168} \frac{7 / x+2880 \int_{0}^{\infty} h^{\prime \prime}(t) e^{-x t} d t}{(1 / x) \int_{0}^{\infty} h^{\prime}(t) e^{-x t} d t}
\end{aligned}
$$

Application of the identity

$$
\frac{1}{x^{n}}=\frac{1}{\Gamma(n)} \int_{0}^{\infty} t^{n-1} e^{-x t} d t \quad \text { for } n>0
$$

and Lemma 1 give (3.7).

Now, to prove $f$ is strictly increasing on $(0, \infty)$, it suffices to prove $t \mapsto A(t) / B(t)$ is increasing on $(0, \infty)$ by Lemma 4 . Similar to the proof of Theorem 1 , we easily see that

$$
\begin{aligned}
& \lim _{t \rightarrow 0} A(t)=\lim _{t \rightarrow 0}\left(7+2880 h^{\prime \prime}(t)\right)=\lim _{t \rightarrow 0}\left[7+2880\left(\frac{1}{t^{2}}-\frac{1}{2 t \sinh (t / 2)}\right)^{\prime \prime}\right]=0, \\
& \lim _{t \rightarrow 0} B(t)=\lim _{t \rightarrow 0}\left(\int_{0}^{t} h^{\prime}(s) d s\right)=0,
\end{aligned}
$$

and the function $A^{\prime} / B^{\prime}=2880 h^{\prime \prime \prime} / h^{\prime}$ is increasing on $\left(0, t_{0}\right)$ and decreasing on $\left(t_{0}, \infty\right)$ by Lemma 7. Then by Lemma 3 it is enough to check that $\operatorname{sgn} B^{\prime}(t) \operatorname{sgn} H_{A, B}(\infty)>0$. In fact, $B^{\prime}(t)=h^{\prime}(t)<0$ for $t>0$ in view of Lemma 7, and

$$
\begin{aligned}
& \lim _{t \rightarrow \infty} \frac{A^{\prime}(t)}{B^{\prime}(t)}=\lim _{t \rightarrow \infty} \frac{2800 h^{\prime \prime \prime}(t)}{h^{\prime}(t)}=0, \\
& \lim _{t \rightarrow \infty} B(t)=\lim _{t \rightarrow \infty} \int_{0}^{t} h^{\prime}(s) d s=h(\infty)-h\left(0^{+}\right)=-\frac{1}{24}, \\
& \lim _{t \rightarrow \infty} A(t)=\lim _{t \rightarrow \infty}\left(7+2880 h^{\prime \prime}(t)\right)=7,
\end{aligned}
$$


which imply that

$$
H_{A, B}(t)=\frac{A^{\prime}(t)}{B^{\prime}(t)} B(t)-A(t) \rightarrow-7 \quad \text { as } t \rightarrow \infty .
$$

This indicates $\operatorname{sgn} B^{\prime}(t) \operatorname{sgn} H_{A, B}(\infty)>0$.

Using the asymptotic formula $[35$, p. $32,(5)]$

$$
\ln \Gamma\left(x+\frac{1}{2}\right)=x \ln x-x+\frac{1}{2} \ln (2 \pi)-\sum_{k=1}^{\infty} \frac{\left(1-2^{1-2 k}\right) B_{2 k}}{2 k(2 k-1)} \frac{1}{x^{2 k-1}}
$$

as $x \rightarrow \infty$, we find that

$$
\begin{aligned}
f(x) & \sim \frac{1}{24 x\left(-\frac{1}{24 x}+\frac{7}{2880 x^{3}}-\frac{31}{40,320 x^{5}}\right)+1}-\frac{120}{7} x^{2} \\
& =\frac{3720}{7} \frac{x^{2}}{98 x^{2}-31} \rightarrow \frac{1860}{343} \quad \text { as } x \rightarrow \infty .
\end{aligned}
$$

While $f\left(0^{+}\right)=1$ is clear. This completes the proof.

\section{Concluding remarks}

Remark 3 In this paper, we investigate the monotonicity of the function $f(x)$. In general, it is difficult to deal with such monotonicity since the gamma function $\Gamma$ occurs in denominator. However, by the aid of Lemma $5, f(x)$ is equivalently changed into the ratio of two Laplace transformations of $A(x)$ and $B(x)$. While Lemma 4 provides exactly an approach to confirm the monotonicity of such ratio. Undoubtedly, it is a novel idea.

Moreover, it is known that Laplace transformation is related to the completely monotonic function. A function $f$ is said to be completely monotonic on an interval $I$ if $f$ has derivatives of all orders on $I$ and satisfies

$$
(-1)^{n} f^{(n)}(x) \geq 0 \quad \text { for all } x \in I \text { and } n=0,1,2, \ldots
$$

If inequality (4.1) is strict, then $f$ is said to be strictly completely monotonic on $I$. The classical Bernstein's theorem $[36,37]$ states that a function $f$ is completely monotonic on $(0, \infty)$ if and only if it is a Laplace transform of some nonnegative measure $\mu$, that is,

$$
f(x)=\int_{0}^{\infty} e^{-x t} d \mu(t)
$$

where $\mu(t)$ is non-decreasing and the integral converges for $0<x<\infty$.

Remark 4 Let $\alpha>\beta$. If $B(t)>0$ for $t>0$ and

$$
\beta<\frac{\int_{0}^{\infty} A(t) e^{-x t} d t}{\int_{0}^{\infty} B(t) e^{-x t} d t}<\alpha,
$$

then, by Bernstein's theorem, both the functions

$$
x \mapsto \int_{0}^{\infty}[A(t)-\beta B(t)] e^{-x t} d t \quad \text { and } \quad x \mapsto \int_{0}^{\infty}[\alpha B(t)-A(t)] e^{-x t} d t
$$


are completely monotonic on $(0, \infty)$. And then, by Theorem 1 , we immediately get the following.

Proposition 1 Both the functions

$$
\begin{aligned}
& g_{1}(x)=2880\left(x^{2}+\frac{31}{98}\right)\left[\ln \Gamma(x+1 / 2)-x \ln x+x-\frac{1}{2} \ln (2 \pi)\right]+120 x+\frac{1517}{49 x}, \\
& g_{2}(x)=-\left(x^{2}+\frac{7}{120}\right)\left[\ln \Gamma(x+1 / 2)-x \ln x+x-\frac{1}{2} \ln (2 \pi)\right]-\frac{1}{24} x
\end{aligned}
$$

are completely monotonic on $(0, \infty)$.

Furthermore, by Bernstein's theorem and Lemma 7, Proposition 1 can be improved as follows.

Theorem 2 The function

$$
g(x)=24 x\left(\frac{120}{7} x^{2}+a\right)[\ln \Gamma(x+1 / 2)-x \ln x+x-\ln \sqrt{2 \pi}]+\frac{120}{7} x^{2}+a-1
$$

is completely monotonic on $(0, \infty)$ if and only if $a \geq 1860 / 343$, and so is $-g(x)$ on $(0, \infty)$ if and only if $a \leq-120 \lambda_{0} / 7 \approx-0.88635$, where $\lambda_{0}$ is defined in Lemma 7 .

Proof By Lemma 5 and identities (3.4), (3.5) and (3.6), $g(x)$ can be written as

$$
\begin{aligned}
g(x) & =-24\left(\frac{120}{7} x^{2}+a\right)\left(\frac{1}{24}+\int_{0}^{\infty} h^{\prime}(t) e^{-x t} d t\right)+\frac{120}{7} x^{2}+a-1 \\
& =-1-\frac{2880}{7} x^{2} \int_{0}^{\infty} h^{\prime}(t) e^{-x t} d t-24 a \int_{0}^{\infty} h^{\prime}(t) e^{-x t} d t \\
& =-24 \int_{0}^{\infty}\left[a-\left(-\frac{120}{7} \frac{h^{\prime \prime \prime}(t)}{h^{\prime}(t)}\right)\right] h^{\prime}(t) e^{-x t} d t
\end{aligned}
$$

Since $h^{\prime}(t)<0$ for $t>0$, by Bernstein's theorem and Lemma 7, $g$ is completely monotonic on $(0, \infty)$ if and only if

$$
a \geq \frac{120}{7} \sup _{t \in(0, \infty)}\left(-\frac{h^{\prime \prime \prime}(t)}{h^{\prime}(t)}\right)=-\frac{120}{7} \inf _{t \in(0, \infty)} \frac{h^{\prime \prime \prime}(t)}{h^{\prime}(t)}=-\frac{120}{7}\left(-\frac{31}{98}\right)=\frac{1860}{343},
$$

and so is $-g$ on $(0, \infty)$ if and only if

$$
a \leq \frac{120}{7} \inf _{t \in(0, \infty)}\left(-\frac{h^{\prime \prime \prime}(t)}{h^{\prime}(t)}\right)=-\frac{120}{7} \sup _{t \in(0, \infty)} \frac{h^{\prime \prime \prime}(t)}{h^{\prime}(t)}=-\frac{120}{7} \lambda_{0} \approx-0.88635
$$

This ends the proof.

Remark 5 The expression of $f(x)$ reminds us to consider the asymptotic expansion of

$$
\frac{1}{24 x(\ln \Gamma(x+1 / 2)-x \ln x+x-\ln \sqrt{2 \pi})+1}:=x^{2} \sum_{n=0}^{\infty} \frac{c_{n}}{x^{2 n}} .
$$


Using asymptotic expansion (3.8), we have

$$
-\left(24 \sum_{n=2}^{\infty} \frac{\left(1-2^{1-2 n}\right) B_{2 n}}{2 n(2 n-1)} \frac{1}{x^{2 n-2}}\right)\left(x^{2} \sum_{n=0}^{\infty} c_{n} x^{-2 n}\right)=1,
$$

that is,

$$
\sum_{n=0}^{\infty}\left(\sum_{k=0}^{n} \frac{\left(1-2^{-2 k-3}\right) B_{2 k+4}}{2(k+2)(2 k+3)} c_{n-k}\right) \frac{1}{x^{2 n}}=-\frac{1}{24} .
$$

Comparing coefficients gives

$$
\begin{aligned}
& -\frac{7}{2880} c_{0}=-\frac{1}{24}, \\
& \sum_{k=0}^{n} \frac{\left(1-2^{-2 k-3}\right) B_{2 k+4}}{2(k+2)(2 k+3)} c_{n-k}=0 \quad \text { for } n \geq 1,
\end{aligned}
$$

which show that $c_{n}$ has the recurrence formula

$$
c_{n}=\frac{2880}{7} \sum_{k=1}^{n} \frac{\left(1-2^{-2 k-3}\right) B_{2 k+4}}{2(k+2)(2 k+3)} c_{n-k} \quad \text { and } \quad c_{0}=\frac{120}{7}
$$

from which we obtain a new asymptotic expansion for the gamma function:

$$
\frac{\Gamma(x+1 / 2)}{\sqrt{2 \pi}(x / e)^{x}} \sim \exp \left(-\frac{1}{24 x}+\frac{1}{24 x^{3}} \frac{1}{\frac{120}{7}+\frac{1860}{343} x^{-2}+\cdots+c_{n} x^{-2 n}+\cdots}\right) \quad \text { as } x \rightarrow \infty .
$$

Moreover, it is easy to prove the inequalities

$$
\begin{aligned}
\exp \left(-\frac{1}{1440} \frac{5880 x^{2}+1517}{x\left(98 x^{2}+31\right)}\right) & =\exp \left(-\frac{1}{24 x}+\frac{1}{24 x^{3}} \frac{1}{\frac{120}{7}+\frac{1860}{343} x^{-2}}\right) \\
& <\frac{\Gamma(x+1 / 2)}{\sqrt{2 \pi}(x / e)^{x}}<\exp \left(-\frac{1}{24 x}+\frac{1}{24 x^{3}} \frac{1}{\frac{120}{7}}\right) \\
& =\exp \left(-\frac{120 x^{2}-7}{2880 x^{3}}\right)
\end{aligned}
$$

hold for $x \geq 1 / 2$.

\section{Acknowledgements}

The authors would like to express their sincere thanks to the editors and reviewers for their great efforts to improve this paper. This work was supported by the Fundamental Research Funds for the Central Universities (No. 2015ZD29) and the Higher School Science Research Funds of Hebei Province of China (No. Z2015137).

\section{Competing interests}

The authors declare that they have no competing interests.

\section{Authors' contributions}

All authors contributed equally to the writing of this paper. All authors read and approved the final manuscript.

\section{Author details}

'College of Science and Technology, North China Electric Power University, Ruixiang Street 282, Baoding, 071051, P.R. China. ${ }^{2}$ Department of Science and Technology, State Grid Zhejiang Electric Power Company Research Institute, Hangzhou, 310014, P.R. China. 


\section{Publisher's Note}

Springer Nature remains neutral with regard to jurisdictional claims in published maps and institutional affiliations.

Received: 30 September 2017 Accepted: 8 December 2017 Published online: 28 December 2017

\section{References}

1. Burnside, W: A rapidly convergent series for log N!. Messenger Math. 46, 157-159 (1917)

2. Gosper, RW: Decision procedure for indefinite hypergeometric summation. Proc. Natl. Acad. Sci. USA 75, 40-42 (1978)

3. Batir, N: Sharp inequalities for factorial $n$. Proyecciones 27(1), 97-102 (2008)

4. Mortici, C: On the generalized Stirling formula. Creative Math. Inform. 19(1), 53-56 (2010)

5. Ramanujan, S: The Lost Notebook and Other Unpublished Papers. Springer, Berlin (1988)

6. Smith, WD: The gamma function revisited. http://schule.bayernport.com/gamma/gamma05.pdf (2006)

7. Nemes, G: New asymptotic expansion for the gamma function. Arch. Math. (Basel) 95, 161-169 (2010)

8. Chen, C-P: A more accurate approximation for the gamma function. J. Number Theory 164, 417-428 (2016)

9. Yang, Z-H, Chu, Y-M: Asymptotic formulas for gamma function with applications. Appl. Math. Comput. 270, 665-680 (2015)

10. Shi, X, Liu, F, Hu, M: A new asymptotic series for the Gamma function. J. Comput. Appl. Math. 195, 134-154 (2006)

11. Guo, B-N, Zhang, Y-J, Qi, F: Refinements and sharpenings of some double inequalities for bounding the gamma function. J. Inequal. Pure Appl. Math. 9(1), Article ID 17 (2008)

12. Batir, N: Inequalities for the gamma function. Arch. Math. 91, 554-563 (2008)

13. Mortici, C: An ultimate extremely accurate formula for approximation of the factorial function. Arch. Math. 93(1), 37-45 (2009)

14. Mortici, C: New sharp inequalities for approximating the factorial function and the digamma functions. Miskolc Math. Notes 11(1), 79-86 (2010)

15. Mortici, C: Improved asymptotic formulas for the gamma function. Comput. Math. Appl. 61, 3364-3369 (2011)

16. Mortici, C: Ramanujan estimate for the gamma function via monotonicity arguments. Ramanujan J. 25(2), 149-154 (2011)

17. Tian, J-F, Ha, M-H: Properties of generalized sharp Hölder's inequalities. J. Math. Inequal. 11(2), 511-525 (2017)

18. Tian, J-F, Ha, M-H: Properties and refinements of Aczél-type inequalities. J. Math. Inequal. 12(1), 175-189 (2018). https://doi.org/10.7153/jmi-2018-12-14

19. Zhao, J-L, Guo, B-N, Qi, F: A refinement of a double inequality for the gamma function. Publ. Math. (Debr.) 80(3-4), 333-342 (2012)

20. Mortici, C: Further improvements of some double inequalities for bounding the gamma function. Math. Comput. Model. 57, 1360-1363 (2013)

21. Feng, L, Wang, W: Two families of approximations for the gamma function. Numer. Algorithms 64, 403-416 (2013)

22. Qi, F: Integral representations and complete monotonicity related to the remainder of Burnside's formula for the gamma function. J. Comput. Appl. Math. 268, 155-167 (2014)

23. Lu, D: A new sharp approximation for the Gamma function related to Burnside's formula. Ramanujan J. 35(1), 121-129 (2014)

24. Mortici, C: A new fast asymptotic series for the gamma function. Ramanujan J. 38(3), 549-559 (2015)

25. Lu, D, Song, L, Ma, C: Some new asymptotic approximations of the gamma function based on Nemes' formula, Ramanujan's formula and Burnside's formula. Appl. Math. Comput. 253, 1-7 (2015)

26. Yang, Z-H, Tian, J: Monotonicity and sharp inequalities related to gamma function. J. Math. Inequal. 12(1), 1-22 (2018). https://doi.org/10.7153/jmi-2018-12-01

27. Alzer, H: On some inequalities for the gamma and psi functions. Math. Comput. 66(217), 373-389 (1997)

28. Yang, Z-H: Approximations for certain hyperbolic functions by partial sums of their Taylor series and completely monotonic functions related to gamma function. J. Math. Anal. Appl. 441, 549-564 (2016)

29. Weisstein, EW: Laplace Transform, From MathWorld - A Wolfram Web Resource. http://mathworld.wolfram.com/LaplaceTransform.html

30. Belzunce, F, Ortega, E, Ruiz, JM: On non-monotonic ageing properties from the Laplace transform, with actuarial applications. Insur. Math. Econ. 40, 1-14 (2007)

31. Yang, Z-H, Chu, Y-M, Wang, M-K: Monotonicity criterion for the quotient of power series with applications. J. Math. Anal. Appl. 428, 587-604 (2015)

32. Xia, F-L, Yang, Z-H, Chu, Y-M: A new proof for the monotonicity criterion of the quotient of two power series on the infinite interval. Pac. J. Appl. Math. 7(2), 97-101 (2015)

33. Yang, Z-H: A new way to prove L'Hospital monotone rules with applications. arXiv:1409.6408 [math.CA]

34. Yang, Z-H: Some properties of the divided difference of psi and polygamma functions. J. Math. Anal. Appl. 455 761-777 (2017). https://doi.org/10.1016/j.jmaa.2017.05.081

35. Luke, YL: The Special Functions and Their Approximations, Vol. I. Academic Press, New York (1969)

36. Bernstein, S: Sur les fonctions absolument monotones. Acta Math. 52, 1-66 (1929)

37. Widder, DV: Necessary and sufficient conditions for the representation of a function as a Laplace integral. Trans. Am. Math. Soc. 33, 851-892 (1931) 